\title{
Rare splenic complications and specific serology: decisive diagnostic tools in two cases of visceral leishmaniasis
}

\author{
Rare complicanze spleniche e diagnosi sierologica: strumenti diagnostici \\ decisivi in due casi di leishmaniosi viscerale
}

\author{
Andrea Celestini $^{a, *}$, Federica Paglia ${ }^{b}$, Orlando Dell' Unto ${ }^{c}$, \\ Riccardo Guarisco ${ }^{c}$, Claudio Puoti ${ }^{c}$
}

\author{
a UOC Cardiologia, Ospedale San Paolo Civitavecchia, Roma \\ b UOC Medicina d'Urgenza, Ospedale Sandro Pertini, Roma \\ ${ }^{\mathrm{C}}$ UOC Medicina, Ospedale San Giuseppe, Marino, Roma
}

Received 19 November 2010; accepted 18 July 2011

available online 13 September 2011

\section{KEYWORDS}

Visceral Leishmaniasis; Splenic infarction; Splenic rupture; Serological tests.

\begin{abstract}
Summary
Introduction: Visceral leishmaniasis (VL) is a major endemic vector-borne disease in Southern Europe. We present two cases of VL, both characterized by splenic complications.

Methods and results: Case 1: A 47-year-old female presented with effort angina, hepatosplenomegaly and pancytopenia. The clinical course was complicated by splenic infarction. Although bone marrow biopsy failed to show amastigotes, diagnosis was performed by a fast agglutinating screening test (FAST) and confirmed by a direct agglutinating test (DAT). The patient was treated successfully with AmBisome. Case 2: A 22-year-old male who had undergone a splenectomy to treat splenic rupture related to a minor trauma four months earlier presented with fever, nocturnal sweats and weight loss. The lack of pancytopenia was due to the absence of the spleen. The first biopsy did not identify parasites, but because the FAST had been positive, another bone marrow biopsy was performed, which demonstrated leishmaniasis. This patient was treated with the same schedule of AmBisome infusion.

Discussion: 1) The clinical presentation of VL can be atypical, 2) splenic complications can characterize this disease, and 3) specific serology may be an important tool to reach a diagnosis. (C) 2011 Elsevier Srl. All rights reserved.
\end{abstract}

* Corresponding author: Via Reggio Calabria 6 - 00161 Roma, Italy.

E-mail: andrea.celestini@gmail.com (A. Celestini). 


\section{Introduction}

Visceral leishmaniasis (VL) or Kala-azar, an infectious disease caused by the bite of Phlebotomus sp., is a major endemic vector-borne disease in Southern Europe. VL affects populations from 70 countries across Southeast Asia, East Africa, South America and the Mediterranean region; the worldwide incidence is estimated to be approximately $8.3 / 100,000$, with approximately 51,000 deaths per year [1]. Although the reported annual incidence in Southern Europe ranges by country from $0.02 / 100,000$ to $0.49 / 100,000$ [1], these numbers are misleading given the number of misdiagnoses and asymptomatic infections. More than 20 pathogenetic species of Leishmania have been identified in the world. Most of these species can cause only the cutaneous form of leishmaniasis; Leishmania infantum is the only agent of $\mathrm{VL}$ in Europe, whereas Leishmania donovani is responsible for $\mathrm{VL}$ in the Indian sub-continent and in Africa [1,2].

After inoculation, the flagellated parasite (promastigote) transforms into an amastigote after being phagocytosed by dermal macrophages, localizing in the macrophagic lysosomes. The initial immune response against VL and the parasite subtype are fundamental to the subsequent development of systemic disease: a Th-1 type response is necessary for a protective reaction, whereas Th-2 differentiation usually induces the development of VL. The incubation period ranges from weeks to months; humans are considered incidental hosts, and dogs are the most frequent animal reservoir [2]. Patients generally exhibit cachexia with recurrent high fever, anorexia and night sweats; hepatosplenomegaly and hypersplenism-related pancytopenia are other common features. VL may coexist with other infectious diseases, and currently, HIV-VL coinfection is an emerging problem in developing countries. Because HIV and Leishmania sp. share the same target immune cells, they exert a synergistic damaging effect on the immune response and may induce atypical clinical manifestations, such as the visceralization of cutaneous leishmaniasis [1].

We report two cases of VL characterized by rare splenic complications.

\section{Case 1}

A 47-year-old Italian HIV-negative female (body weight $52 \mathrm{~kg}$ ), who lived in a farm in South Latium, was admitted to our department presenting with wasting and anorexia over the last month, accompanied in the last two weeks by effort-related chest pain. No history of smoking or alcohol abuse was reported. Physical examination showed significant hepatosplenomegaly without appreciable lymphoadenopathy. Laboratory values revealed pancytopenia with a prevalent reduction in red blood cells and polyclonal hypergammaglobulinemia (for more details, see Table 1). A chest $\mathrm{X}$-ray, an ECG and a cardiac ultrasound were normal. During hospitalization, epigastric tenderness developed, and the patient underwent an abdominal ultrasound that revealed a sub-capsular hypoechogenic area around the inferior wall of the spleen. This sign was confirmed by magnetic resonance imaging, which showed intrasplenic hyperintensity, consistent with a hemorrhagic infarction. No acquired (antiphospholipid antibodies, oral contraceptives) or congenital thrombophilic factors (factor $\mathrm{V}$ leiden, prothrombin G20210A or methylenetetrahydrofolate reductase mutations or a lack of antithrombin III, protein C or S) were evidenced. Moreover, tests for viral hepatitis markers, the Wright test and the tine test were negative. Although peripheral blood and bone marrow specimens failed to show amastigotes, a fast agglutinating screening test (FAST) and a direct agglutinating test for Leishmania infantum (DAT) (dilution of 1:4000) revealed the presence of anti-Leishmania antibodies. A splenic fine needle aspirate sample (FNAS) was not taken because taking such a sample was considered dangerous in the presence of a splenic hemorrhage, and polymerase chain reaction (PCR) was unavailable in our hospital. The patient was treated with liposomal amphotericin $\mathrm{B}(\mathrm{AmBi}$ some) $(4 \mathrm{mg} / \mathrm{kg}$ i.v for 5 days and on days 14 and 21). After treatment, a progressive improvement of the patient's anemic status and abdominal symptoms was observed.

\section{Case 2}

A 22-year-old Albanian male (body weight $72 \mathrm{~kg}$ ), who had worked in Greece for a while and then had moved to Italy (South Latium), was admitted to our department. The patient, who had no history of alcohol abuse, presented with asthenia, weight loss, irregular fever and night sweats. Four months earlier, the patient had undergone a splenectomy to treat a splenic rupture resulting from a minor trauma. Macroscopic examination of the spleen had documented marked splenomegaly $(21 \times 19 \times 7 \mathrm{~cm})$ and lymphadenomegaly of the splenic hilum $(2.2 \mathrm{~cm})$. The histological diagnosis was primary hypersplenism.

Laboratory tests showed normal levels of platelets and white cells, slight anemia and increased levels of hepatolysis markers (for more details, see Table 2). Hepatotropic

Table 1 Case 1: lab values before and after treatment.

\begin{tabular}{|c|c|c|c|c|c|c|c|c|c|}
\hline & date & $\begin{array}{c}\text { r.b.c. } \\
\left(\times 10^{6} / \mu l\right)\end{array}$ & $\begin{array}{l}\text { w.b.c. } \\
\left(\times 10^{3} / \mu l\right)\end{array}$ & $\begin{array}{l}\text { hemoglobin } \\
\qquad(\mathrm{g} / \mathrm{dl})\end{array}$ & $\begin{array}{c}\text { hematocrit } \\
\text { (\%) }\end{array}$ & $\begin{array}{l}\text { platelets } \\
\left(\times 10^{3} / \mu \mathrm{l}\right)\end{array}$ & $\begin{array}{c}\text { iron } \\
(\mu g / d l)\end{array}$ & $\begin{array}{l}\text { proteins } \\
(\mathrm{g} / \mathrm{dl})\end{array}$ & $\begin{array}{l}\text { albumin } \\
\text { (g/dl) }\end{array}$ \\
\hline Before treatment & April, $28^{\text {th }}$ & 3.60 & 2.00 & 9.30 & 26.0 & 112 & 24 & 5.4 & 2.8 \\
\hline Before treatment & May, $05^{\text {th }}$ & 3.10 & 2.00 & 7.60 & 23.0 & 77 & & & \\
\hline After treatment & June, $09^{\text {th }}$ & 3.49 & 2.29 & 9.26 & 26.8 & 80 & & & \\
\hline After treatment & June, $14^{\text {th }}$ & 3.61 & 2.50 & 9.70 & 32.2 & 87 & & 6.1 & 3.57 \\
\hline After treatment & June, $25^{\text {th }}$ & 4.40 & 2.80 & 11.70 & 34.8 & 189 & 75 & & \\
\hline After treatment & July, $05^{\text {th }}$ & 4.46 & 3.03 & 12.00 & 34.8 & 161 & 70 & 7.2 & 4.3 \\
\hline
\end{tabular}

Legend: r.b.c.: red blood cells, w.b.c: white blood cells. 
Table 2 Case 1: lab values before and after treatment.

\begin{tabular}{|c|c|c|c|c|c|c|c|c|c|c|}
\hline & date & $\begin{array}{c}\text { r.b.c. } \\
\left(\times 10^{6} / \mu l\right)\end{array}$ & $\begin{array}{c}\text { w.b.c. } \\
\left(\times 10^{3} / \mu l\right)\end{array}$ & $\begin{array}{l}\text { hemoglobin } \\
(\mathrm{g} / \mathrm{dl})\end{array}$ & $\begin{array}{c}\text { hematocrit } \\
\text { (\%) }\end{array}$ & $\begin{array}{l}\text { platelets } \\
\left(\times 10^{3} / \mu \mathrm{l}\right)\end{array}$ & $\begin{array}{l}\text { iron } \\
(\mu \mathrm{g} / \mathrm{dl})\end{array}$ & $\begin{array}{l}\text { AST } \\
(\mathrm{U} / \mathrm{l})\end{array}$ & $\begin{array}{c}\mathrm{ALT} \\
(\mathrm{U} / \mathrm{l})\end{array}$ & $\begin{array}{c}\text { total } \\
\text { bilirubin } \\
\text { (mg/dl) }\end{array}$ \\
\hline Before treatment & October, $9^{\text {th }}$ & 4.98 & 8.6 & 12.5 & 37 & 337 & 43 & 325 & 280 & 1.34 \\
\hline Before treatment & November, $21^{\text {st }}$ & 4.25 & 11.8 & 11 & 33.7 & 754 & 36 & 19 & 30 & 0.42 \\
\hline After treatment & January $15^{\text {th }}$ & 5.40 & 9.7 & 14 & 38 & 437 & 60 & 37 & 34 & 0.33 \\
\hline
\end{tabular}

Legend: r.b.c.: red blood cells, w.b.c: white blood cells, AST: aspartate aminotrasferase, ALT: alanine aminotrasferase.

infections (HAV, HBV, HCV, HBV, CMV) were excluded by serological testing. HIV, brucellosis, toxoplasmosis, rickettsiosis and typhoid and paratyphoid fevers were also excluded. Serial hemocultures were negative.

A chest X-ray and echocardiography were normal. Abdominal ultrasound revealed liver enlargement with extreme lymphadenomegaly $(1.5 \mathrm{~cm})$ at the hepatic hilum and at the interaortocaval chain. A peripheral blood smear and a bone marrow biopsy were negative, while FAST confirmed the suspected clinical diagnosis of VL. We decided to perform a second bone marrow biopsy that finally showed amastigotes. As in the first case, the patient was treated with AmBisome ( $4 \mathrm{mg} / \mathrm{kg}$ i.v for 5 days and on days 14 and 21).

\section{Discussion}

The diagnosis of VL is generally difficult because fever, splenomegaly and pancytopenia can be caused by a wide range of infectious, hematological or systemic disorders.

The clinical cases above reported showed the following: 1) peculiar clinical manifestations, 2) uncommon complications, 3) unusual methods to reach the final diagnosis and 4) successful treatment with AmBisome.

1) The first patient presented without fever or sweating but with chest pain suggestive of an anemia-related angina pectoris. However, the concomitant presence of important spleen enlargement and severe pancytopenia helped us to reach the final diagnosis. Endocarditis was not considered because of the lack of fever and the absence of valvular abnormality on the echocardiogram. The lack of relevant alterations in the peripheral and bone marow specimens excluded hematological disorders, and the tine test and chest $\mathrm{X}$-ray excluded tuberculosis. The abdominal ultrasound, serological markers and the negative anamnesis for alcohol abuse ruled out cirrhotic involvement of the liver. The second patient presented with typical symptoms of VL (asthenia, weight loss, irregular fever and night sweats during the previous month), but the absence of spleen masked the typical hypersplenism-induced pancytopenia pattern of VL. The first clinical suspect was hepatitis, due to the abnormal serum alanine aminotraferase (ALT) levels, but hepatitis was excluded by serological procedures. Hematological diseases, considered because of the diffuse abdominal adenopathy, were ruled out by the peripheral and bone marrow smears. Cardiac ultrasound and hemocultures excluded endocarditis, and melitensis, rickettsiosis and typhoid and paratyphoid fevers were ruled out by agglutination tests.

2) In the first case, the clinical course was complicated by a hemorrhagic spleen infarction. This spleen complication, to date not reported during VL, is usually found in patients with endocarditis or portal vein thrombosis and is generally related to embolisms, which that were excluded in our case. Although a VL-related splenic infarction has never been reported in the literature, it is known to be a rare complication of other parasite infestations such as malaria. In VL, the microvascular sequestration of parasitized red blood cells and the ischemia induced by the hyperplasia of the reticuloendothelial system seem to be the factors allowing the onset of splenic infarction. It is likely that VL shares the same predisposing factors of malaria in the development of splenic infarction $[3,4]$

In the second case, the past splenomegaly was likely a clinical manifestation of VL, which lasted for long time, and the rupture of the spleen after a minor trauma is an extremely rare complication of VL-induced hypersplenism; to the best of our knowledge, only one other case of nearly spontaneous splenic rupture following VL has discussed in literature to date [5].

3) Another important issue is that serological diagnosis was important in both cases.

Direct observation of parasites is the gold standard of VL diagnosis; however, peripheral blood is usually negative, even during the acute phase of $\mathrm{VL}$, and the detection using bone marrow specimens is characterized by low sensitivity. Therefore, it may be helpful to obtain a second bone marrow sample or to perform a spleen FNAS or a hepatic biopsy (when lab markers suggest important hepatic involvement) $[6,7]$. In the first case, the peripheral blood smear and the bone marrow biopsy failed to detected amastigotes. FNAS of the spleen was not performed because it was considered potentially harmful, and a hepatic biopsy was not performed because of the absence of serological evidence of hepatitis (i.e., the patient had normal ALT levels). However, on the basis of the clinical context, pharmacological treatment was initiated, and because the clinical and biochemical patterns progressively improved, the diagnosis was confirmed ex juvantibus. In the second case, the identification of amastigotes only confirmed the diagnosis because they were observed in the second bone marrow sample, after the FAST had been performed.

Serological tests have been progressively replaced the direct identification of parasites for the diagnosis of $\mathrm{VL}$ [8]. The agglutination tests (DAT and FAST) use microplates in which the blood of the patient is mixed with stained parasites or parasite antigens. In the presence of antibodies against Leishmania, agglutination occurs. Agglutination tests require different antigens because of the different species of Leishmania; the predictivity of these tests depends on type of parasite prevalent in that area. 
For example, for Leishmania donovani, which is found in developing countries, the DAT has high sensitivity and specificity $(95.9 \%$ and $99.4 \%$, respectively, at a $1: 4000$ dilution) [9], while the DAT for Leishmania infantum has a similar sensitivity (95.4\%) but a lower specificity (88.5\%) at a 1:1600 dilution [9]. In contrast to the DAT, the FAST, characterized by similar predictive values, makes use of only one serum dilution, and the results can be read sooner (within $3 \mathrm{~h}$, as opposed to $18-20 \mathrm{~h}$ for the DAT) [10,11]. As shown in this report, agglutination tests represent a good tool in the diagnosis of VL, especially when, in a high suspicious context, parasites have not been visualized. In the past several years, serological tests using recombinant Leishmania antigens (rK39 or rK26: recombinant antigens derived from 39- and 26-amino-acid repeats, respectively) have been developed. These antigens are used in enzymelinked immunosorbent assays. Although the tests using rK26 seem to have a low sensitivity, thus limiting their use, rK39 assays have a high sensitivity and specificity, comparable to those of DAT. These characteristics are gradually making rK39 assays the most appropriate serological tests for the diagnosis of VL $[1,8]$. Indirect immunofluorescence assays are commonly used for the detection of anti-Leishmania antibodies, but the sensitivity is not well defined, ranging between 28.5 and $86.6 \%$ depending on the study. Recently, PCR was shown to be a highly specific and highly sensitive technique [12]; however, despite its reproducibility, PCR is expensive and is not yet available in most hospitals.

4) We treated patients with AmBisome, which requires infusion for only a few days and seems to produce fewer adverse reactions than traditional drugs. Since the introduction of pentavalent antimonial agents in the 1940s, various anti-parasite agents have been successfully used against leishmaniasis; however, which is the best therapeutic choice in term of efficacy, side effects and costs is still a matter of debate. Currently, the treatment options for VL include antimonials (sodium stibogluconate and glucantime), the aminoglycoside paromomycin, the phosphocholine analogue miltefosine, amphotericin B deoxycholate and liposomal amphotericin B (AmBisome) $(1,13,14)$. All drugs are given parenterally with the exception of miltefosine (oral administration). The use of antimonials is limited because of their severe side effects (dilated cardiopathy or chronic pancreatitis), the long period of parenteral therapy and drug resistance. Miltefosine, paromomycin and amphotericin $\mathrm{B}$ also require a long treatment periods (from three to four weeks), produce relevant side effects and have contraindications $[1,13,14]$. In contrast, AmBisome, which was approved in the US in 1997, is a short-course therapy with high efficacy and is generally well tolerated. The primary limitation of this therapy is the high cost; however, in the last several years, a new preferential price for developing countries has been introduced $[15,16]$. Both cases reported herein were treated with a five day infusion $(4 \mathrm{mg} / \mathrm{kg})$ and then single infusions during the second and third weeks. New promising data suggest that a single infusion of $10 \mathrm{mg} / \mathrm{kg}$ is not inferior to conventional therapy with amphotericin B deoxycholate [16]. Finally, good results seem to come from combination therapies that include a single dose of
AmBisome followed by seven days of miltefosine, AmBisome (single dose) plus paromomycin for ten days or a full dose of paromomycin and miltefosine for ten days $[1,15]$.

These case reports highlight that 1) the clinical presentation of VL can be atypical, 2) spleen complications can characterize the clinical course of this infection, and 3) immunological techniques may be important diagnostic tools when traditional methods fail to reveal parasites or PCR is not available.

\section{Conflict of interest}

The authors have no conflict of interest.

\section{References}

[1] Mondal S, Bhattacharya P, Ali N. Current diagnosis and treatment of visceral leishmaniasis. Expert Rev Anti Infect Ther 2010;8:919-24.

[2] Herwald BL. Leishmaniasis. Lancet 1999;354:1191-9.

[3] Cinquetti G, Banal F, Rondel C, Plancade D, de Saint Roman C, Adriamanantena $D$, et al. Splenic infarction during Plasmodium ovale acute malaria: first case reported. Malar J 2010;18:288.

[4] Kim A, Park YK, Lee JS, Chung MH, Kim ES. A case of symptomatic splenic infarction in vivax malaria. Korean J Parasitol 2007;45:55-8.

[5] Rovira RE, Díaz-Gómez JR, Lapuebla X, Aquar MC. Spontaneous rupture of the spleen in a patient with visceral leishmaniasis. Enferm Infecc Microbiol Clin 2005;23:327.

[6] Artan R, Yilmaz A, Akçam M, Aksoy NH. Liver biopsy in the diagnosis of visceral leishmaniasis. J Gastroenterol Hepatol 2006;21:299-301.

[7] Chulay JD, Bryceson AD. Quantitation of amastigotes of Leishmania donovani in smears of splenic aspirates from patients with visceral leishmaniasis. Am J Trop Med Hyg 1983;32:475-9.

[8] Chappuis F, Rijal S, Soto A, Menten J, Boelaert M. A meta-analysis of the diagnostic performance of the direct agglutination test and rK39 dipstick for visceral leishmaniasis. BMJ 2006;333:723.

[9] Boelaert M, El Safi S, Jacquet D, de Muynck A, van der Stuyft P, Le Ray D. Operational validation of the Direct Agglutination Test for diagnosis of visceral leishmaniasis. Am J Trop Med Hyg 1999;60:129-34.

[10] Akhoundi B, Mohebali M, Babakhan L, Edrissian GH, Eslami MB, Keshavarz $\mathrm{H}$, et al. Rapid detection of human Leishmania infantum infection: a comparative field study using the fast agglutination screening test and the direct agglutination test. Journal Travel Medicine and Infectious Disease 2010;8:305-10.

[11] Schoone GJ, Hailu A, Kroon CC, Nieuwenhuys JL, Schallig HD, Oskam L, et al. A fast agglutination screening test (FAST) for the detection of anti-Leishmania antibodies. Trans R Soc Trop Med Hyg 2001;95:400-1.

[12] van der Meide W, Guerra J. Comparison between quantitative nucleic acid sequence-based amplification, real-time reverse transcriptase PCR, and real-time PCR for quantification of Leishmania parasites. J Clin Microbiol 2008;46:73-8.

[13] Maltezou HC. Visceral leishmaniasis: advances in treatment. Recent Pat Antiinfect Discov 2008;3:192-8.

[14] Treatment of visceral leishmaniasis in 2010: direction from Bihar State, India. Murray HW. Future Microbiol 2010;5:1301-3.

[15] Sundar S, Chakravarty J. Liposomal amphotericin B and leishmaniasis: dose and response. J Glob Infect Dis 2010;2:159-66.

[16] Sundar S, Chakravarty J, Agarwal D, Rai M, Murray HW. Singledose liposomal amphotericin B for visceral leishmaniasis in India. N Engl J Med 2010;362:504-12. 